\title{
Olivier RAMPIN
}

\author{
Laboratoire de Neurobiologie des Fonctions \\ Végétatives - UR 1060 \\ INRA, Bât. 325, Jouy-en-Josas
}

\section{Men in committed, romantic relationships have lower testosterone. Hormones and Behavior, 2003, 44 : 119-122.

\author{
Burnham T.C., Flynn Chapman J., Gray P.B., \\ McIntyre M.H., Lipson S.F., Ellison P.T.
}

Chez l'homme, les variations des taux de testostérone peuvent interagir avec le comportement sexuel et le comportement parental. Selon l'hypothèse que les auteurs souhaitent tester, les hommes qui entretiennent une relation durable avec une partenaire présenteraient une testostéronémie plus faible que celle d'hommes n'ayant pas un tel mode de vie. Pour vérifier leur hypothèse, les auteurs ont mesuré les quantités de testostérone présente dans des échantillons de salive prélevée (entre $10 \mathrm{H}$ et $10 \mathrm{H} 20$ le matin durant 9 jours consécutifs) chez 122 étudiants (âgés de 27 à 29 ans) de l'Ecole de Commerce de Harvard. Les étudiants étaient payés (10 dollars US). Trente quatre étudiants étaient mariés et sans enfant, 9 étaient mariés avec enfants, trente-huit étudiants non mariés entretenaient une relation stable avec une partenaire, enfin 41 étudiants n'avaient pas de relation stable. Les taux de testostérone salivaires étaient de $250 \mathrm{pmol} / \mathrm{L}$ chez les étudiants mariés et parents, ils étaient compris entre 300 et $350 \mathrm{pmol} / \mathrm{L}$ chez les étudiants mariés ou non et sans enfant mais vivant en couple stable, enfin ils étaient de $400 \mathrm{pmol} / \mathrm{L}$ chez les étudiants ne vivant pas en couple. Les taux de testostérone des étudiants vivant en couple sont significativement inférieurs de $21 \%$ à ceux mesurés chez les célibataires. Les étudiants qui sont père ont des taux de testostérone inférieurs de $41 \%$ à ceux des étudiants qui ne vivent pas de relation stable avec une partenaire, et inférieurs de $28 \%$ à ceux des étudiants vivant en couples mariés ou non et sans enfant.

Malgré les biais expérimentaux représentés par un faible échantillonnage et une population très homogène en âge et en milieu socioculturel, l'étude apporte des arguments en faveur d'une relation entre testostérone, comportement sexuel et comportement parental. Elle confirme l'hypothèse du "défi », selon laquelle des taux élevés de testostérone facilitent la compétition entre mâles pour la reproduc- tion. Les auteurs ne différencient pas la cause des effets : selon eux, les interactions entre testostérone et comportements sont réciproques. Par exemple, les hommes à forte testostéronémie sont peut-être moins enclins à construire des relations stables avec une partenaire. A l'inverse, l'établissement de relations stables pourrait déclencher une chute de la testostéronémie, ce qui aurait pour conséquence de diminuer les tentatives de relations extra-conjugales. Seules les études longitudinales peuvent apporter des réponses claires à ces différents points. Les auteurs poussent plus loin leur raisonnement en rappelant les conséquences des testostéronémies élevées : si celles-ci sont en effet un facteur favorisant la prise de risque, elles sont également corrélées avec une altération des fonctions immunitaires, et donc auraient des effets délétères pour l'individu. Les auteurs concluent en retenant que les relations stables avec une partenaire sont un facteur prédictif des taux de testostérone beaucoup plus fiable que le statut marital.

\section{Impact of single neonatal serotonin treatment (hormonal imprinting) on the brain serotonin content and sexual behavior of adult rats. Life Sciences, 2003, $73:$ 2703-2711.}

\section{Csaba G., Knippel B., Karabelyos C., Inczefi-Gonda} A., Hantos M., Tekes K.

Une maturation des récepteurs des hormones accompagne la différenciation cellulaire. La période périnatale est la période critique de cette maturation. A ce moment-là, la première rencontre du récepteur avec son ligand endogène, l'hormone, crée une empreinte hormonale qui est nécessaire à l'achèvement de la maturation du récepteur. Cependant, en présence d'un excès d'hormone endogène, ou d'autres molécules capables de se lier au récepteur (hormones de la même famille, hormones de synthèse, poliuants environnementaux, etc...) le récepteur développe une empreinte hormonale anormale qui aura des conséquences durant la vie de l'individu sur l'affinité du récepteur pour son ligand, sur son comportement, etc... Les auteurs proposent de rechercher de telles modifica- 
tions chez des rats mâles et femelles soumis à une seule exposition à la sérotonine le jour de leur naissance i) sur les taux de sérotonine cérébrale, ii) sur le comportement sexuel et iii) sur l'affinité de deux récepteurs pour leur ligand : le récepteur des glucocorticoïdes dans le thymus et le récepteur des oestrogènes dans l'utérus.

Les jeunes rats mâles adultes qui ont reçu une seule injection de sérotonine le jour de leur naissance sont plus actifs sexuellement que leurs congénères non traités. Ceci se manifeste par un plus grand nombre d'animaux éjaculant durant un test d'accouplement avec une femelle, et seuls les mâles traités présentent plusieurs éjaculations durant un test de durée limitée à 30 minutes (différences significatives). En revanche la sérotonine n'exerce pas un tel effet activateur du comportement sexuel chez les femelles traitées.

Le traitement néonatal par la sérotonine abaisse significativement les quantités de sérotonine mesurées dans le striatum des jeunes mâles. II ne modifie pas l'affinité du récepteur des glucocorticoïdes ni celle du récepteur des œstrogènes pour leur ligand.

Les résultats montrent un impact du traitement néonatal par la sérotonine sur le comportement sexuel du rat mâle et sur la quantité de sérotonine dans le striatum des mêmes sujets. Le rôle du striatum dans l'excitation sexuelle a été démontré chez le rat, et la présence d'une femelle augmente la synthèse de sérotonine par les terminaisons nerveuses sérotoninergiques innervant cette structure. L'effet néonatal est sexe-dépendant, puisqu'il ne touche pas les femelles.

D'une façon plus générale, cet article pose le problème considérable de l'impact de molécules endogènes et exogènes sur l'avenir de lignées cellulaires lorsque celles-ci se différencient, et lorsqu'elles sont extrêmement sensibles à leur environnement.

\section{Estradiol in the male rat amygdala facilitates mounting but not ejaculation. Physiology and Behavior, 2003, 79: 239-246.}

\section{Huddleston G.G., Michael R.P., Zumpe D., Clancy A.N}

Plusieurs noyaux du système nerveux central tels que l'amydgale participent au contrôle du comportement sexuel chez le rat mâle. Les montes, les intromissions et les éjaculations sont les événements comportementaux que les expérimentateurs utilisent pour quantifier le comportement sexuel du mâle dans cette espèce. Les neurones de l'amygdale portent des récepteurs des oestrogènes, et l'on a démontré que ces neurones sont excités durant l'accouplement. L'injection de Fadrozole (letrozole, un inhibiteur non stéroïdien de l'aromatase, l'enzyme responsable de la synthèse d'oestradiol à partir de testostérone) dans l'amydgale déprime fortement le comportement sexuel chez le rat mâle. Pour rechercher un rôle spécifique de l'oestradiol dans le contrôle du comportement sexuel par l'amygdale, les auteurs ont traité deux groupes de rats sexuellement entraînés par le Fadrozole délivré par voie générale, déprimant ainsi la synthèse d'oestradiol dans l'ensemble du cerveau. Ils supplémentent un des deux groupes traités par le Fadrozole par des injections d'oestradiol dans l'amygdale. Dans ce groupe, les rats ne présentent pas de chute de leur activité sexuelle, ni du nombre des montes et des intromissions durant l'accouplement. En revanche le nombre des éjaculations diminue significativement. Dans le groupe de rats traités par le Fadrozole mais non supplémentés par l'oestradiol, les auteurs enregistrent une chute considérable du nombre d'animaux sexuellement actifs, et par conséquent du nombre des montes, des intromissions et des éjaculations.

Les résultats confirment le rôle important de l'oestradiol dans le contrôle du comportement sexuel par le système nerveux central chez le mâle. Ils identifient l'amygdale comme un acteur important de ce contrôle. Ils rappellent enfin que les différentes séquences du comportement sexuel, au moins chez le rat, sont placées sous le contrôle de différents noyaux du système nerveux central. 